\title{
Muscle ridge between atrioventricular valves and malalignment of junction of these valves with ventricular septum
}

\author{
ANNE DEVLOO-BLANCQUAERT AND DONALD G. RITTER \\ From Rijksuniversiteit, Gent, Belgium; and Mayo Clinic and Mayo Foundation, Rochester, \\ Minnesota 55901, USA
}

SUMMARY A posteromedian muscle ridge is found in the left ventricle between the two atrioventricular valves in congenital malformations of the heart, where malalignment exists between the ventricular septum and the junction of both atrioventricular valve rings; this is seen in transposition with straddling tricuspid valve and double inlet ventricle with outflow chamber. In hearts with normal alignment of the atrioventricular valve and transposition of the great vessels, the same posteromedian muscle ridge was identified in all specimens with associated posterior ventricular septal defect without a muscular rim between the tricuspid valve and the edge of the ventricular septal defect. If this rim was present or if the ventricular septal defect was midseptal, only 50 per cent of the specimens studied showed a posteromedian muscle ridge. This muscle ridge was present in only 20 per cent of the hearts without a ventricular septal defect or with an anterior ventricular septal defect.

The posteromedian muscle ridge has a narrow cranial part (head) which is just apical from the junction of the atrioventricular valve rings or apical from the septum membranaceum or its remnants. The middle part (body) is wider and occasionally double, extending down the posteromedian side of the anterior leaflet of the mitral valve, on the dorsal free wall of the left ventricle, or in the angle between those two structures. It then branches out and joins the base of the posterior papillary muscle and an accessory papillary muscle, which holds the left ventricular part of the tricuspid valve.

The specimens studied showed a full range of malformations from uncomplicated transpositions, wherein the muscle ridge cannot or can scarcely be identified, to varying degrees of straddling tricuspid valve, wherein the valve moves further and further away from the septum, and finally to the double inlet ventricle of the left ventricular type, wherein the bulboventricular septum lies at the opposite side of the right atrioventricular valve.

In double inlet ventricle of the right ventricular type with left posterior pouch, both atrioventricular valves and the posteromedian muscle ridge crossed the septum and were found in the right ventricle.

In studying the left ventricle in transposition of the great arteries of the heart with straddling right atrioventricular valves and in double inlet ventricle, we noticed a large muscle ridge distal to the junction of the atrioventricular valve rings.

In published reports the attachments of the chordae tendineae of both atrioventricular valves have been described at length in cases of straddling tricuspid valve (Mehrizi et al., 1966; De la Cruz and Miller, 1968; Rastelli et al., 1968; Liberthson et al., 1971; Tandon et al., 1974). Little attention

Received for publication 7 December 1977 has been paid, however, to the relation of the valve annuli and the muscle ridge that separates them. Liberthson et al. (1971) described the condition thus: 'A median ridge either independent of or fused with the accessory posterior papillary muscle of the left ventricle often extended from the central fibrous body to the apex, on the posterior wall.' Recently Wenink et al. (1976) mentioned the presence of such a ridge in 35 per cent of transpositions without ventricular septal defect, in 80 per cent of transpositions with ventricular septal defect, and in 3.5 per cent of normal hearts.

The purposes of this study are (1) to describe in 
Table Diagnosis of 137 specimens studied

\begin{tabular}{|c|c|c|}
\hline Diagnosis & Specimens & $\begin{array}{l}\text { Per cent with } \\
\text { PMMR }\end{array}$ \\
\hline \multirow[t]{2}{*}{96 Transpositions } & $\begin{array}{l}22 \text { with intact ventricular septum } \\
16 \text { with anterior ventricular septal defect } \\
58 \text { with posterior or midseptal ventricular septal detect }\end{array}$ & $\begin{array}{l}18 \\
25\end{array}$ \\
\hline & $\begin{array}{l}36 \text { with muscular rim between tricuspid valve and edges of } \\
\text { ventricular septal defect } \\
22 \text { without muscle rim }\end{array}$ & 50 \\
\hline \multirow[t]{3}{*}{13 Double inlet ventricle } & 10 with left ventricular morphology and anterior outflow chamber & \\
\hline & $\begin{array}{l}7 \text { with } 2 \text { atrioventricular valves } \\
3 \text { with common atrioventricular valve }\end{array}$ & $\begin{array}{c}100 \\
? \\
100\end{array}$ \\
\hline & $\begin{array}{l}3 \text { with right ventricular morphology } \\
2 \text { with left posterior pouch } \\
1 \text { without pouch }\end{array}$ & 100 \\
\hline 28 Complete common atrioventricular canal & & 0 \\
\hline
\end{tabular}

detail the posteromedian muscle ridge, (2) to define its presence and position in different types of transposition, in double inlet ventricle of the left and of the right morphological type, and in complete atrioventricular canal, and (3) to study the spectrum of straddling tricuspid valve.

\section{Material and definitions}

Ninety-six specimens of transposition of the great arteries, all from the Mayo Clinic, were studied (Table). In these specimens the ventricular septal defect was essentially a nonjunction between the infundibular septum and the ventricular septum (Goor and Edwards, 1973). The ventricular septal defect may extend posteriorly, anteriorly, or in both directions. When it extends posteriorly, the membranous septum is partly or completely missing and the muscular rim between the edge of the defect and the tricuspid valve is not always present. If this rim is absent, it is sometimes difficult to determine whether or not the tricuspid valve is straddling the septum.

In our study we grouped all defects extending posteriorly under the heading 'posterior ventricular septal defect.' In some cases the defect was situated in the middle of the muscular septum away from the valve annuli and was referred to as 'midseptal ventricular septal defect.' Infrequently the defect was exclusively anterior, sometimes extending into the infundibular septum. In such cases we used the term 'anterior ventricular septal defect'.

Thirteen specimens with double inlet ventricle (9 from the Mayo Clinic and 4 from Gent, Belgium) were also analysed. In 10 of them the ventricle had a left ventricular morphology and an anterior outlet chamber. Three double inlet ventricles showed right ventricular morphology and 2 of these had a small left posterior chamber with no inflow or outflow portion and a smooth septal surface. This pouch, however, did communicate with the large ventricle by way of a small septal defect (Table).

In addition a study was made of 28 hearts with a common atrioventricular canal ( 2 from the Mayo Clinic, 21 from Leiden, Holland, and 5 from Gent) (Table).

\section{Results and description}

In 4 of 10 specimens with posterior ventricular septal defect, with varying degrees of straddling of the tricuspid valve over the ventricular septum, the morphological right atrium was connected by a straddling tricuspid valve to a right-sided chamber with characteristics of the right ventricle, and a left atrium was connected by a mitral valve with a leftsided chamber with characteristics of the left ventricle (normally situated ventricles). All of these specimens had right-sided transposed aortas.

In the other 6 of these 10 specimens, the chamber with characteristics of the right ventricle was to the left of the chamber with left ventricular characteristics (ventricular inversion). In such hearts, the left atrioventricular valve straddles the septum, having characteristics of the tricuspid valve, and the transposed aorta is on the left side. The atrioventricular valve rings meet at the central fibrous body and are attached to it, the atrial septum being attached to the same structure. The ventricular septum does not join the central fibrous body in cases with straddling tricuspid valve but is more to the right in normally situated ventricles (Fig. 1) and to the left if the ventricles are inverted (Fig. $2 \mathrm{~A}$ ). Just distal to the junction of both atrioventricular valve rings, a muscular structure protrudes into the left ventricular cavity (Fig. 1 and 2 A). This structure extends down the posterior wall of the left ventricle towards the apex and joins the posterior papillary 


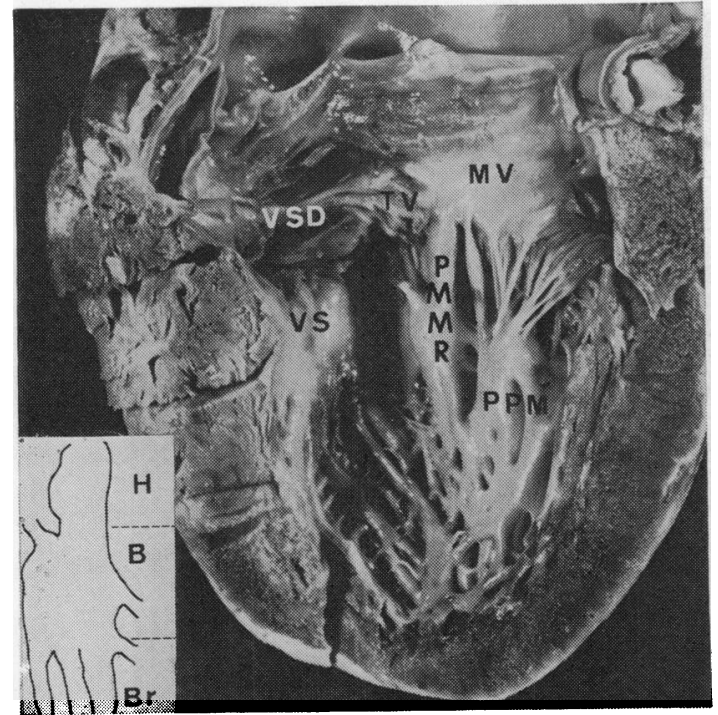

Fig. 1 Transposition with aorta to right and anterior to pulmonary artery, posterior ventricular septal defect (VSD), and straddling tricuspid valve (TV): VSD to left; in middle, under junction of mitral valve (MV) and (TV), a posteromedian muscle ridge (PMMR), with narrow head $(H)$, wider body $(B)$, and branches $(B r)$ (see inset) joining posterior papillary muscle (PPM) of ventricle. muscle of the left ventricle (Fig. 1). If an accessory or abnormal papillary muscle is present in the left ventricle to hold the chordae of the left ventricular part of the straddling tricuspid valve, the caudal end of the posteromedian muscle ridge extends between this accessory papillary muscle and the posterior papillary muscle and often has connections with both (Fig. $2 \mathrm{~A}$ ).

Usually the posteromedian muscle ridge is narrow at its cranial end, known as 'the head' (Fig. 1), becomes wider and more protruding in its middle portion (the body), and then divides into several muscle bundles (branches), which become progressively smaller, finally appearing like small trabeculae at the site where they join the papillary muscles. Sometimes the accessory papillary muscle joins the median muscle already at its middle portion (Fig. 1). This middle portion is often double (Fig. $3 \mathrm{~A}$ ).

When there is an extensive overriding of the tricuspid valve and the junction of both atrioventricular valve rings is displaced far away from the septum, this muscle is completely dissociated from the ventricular septum (Fig. 1). When the tricuspid valve is not straddling or is only slightly straddling, this muscle seems to glide off the septum
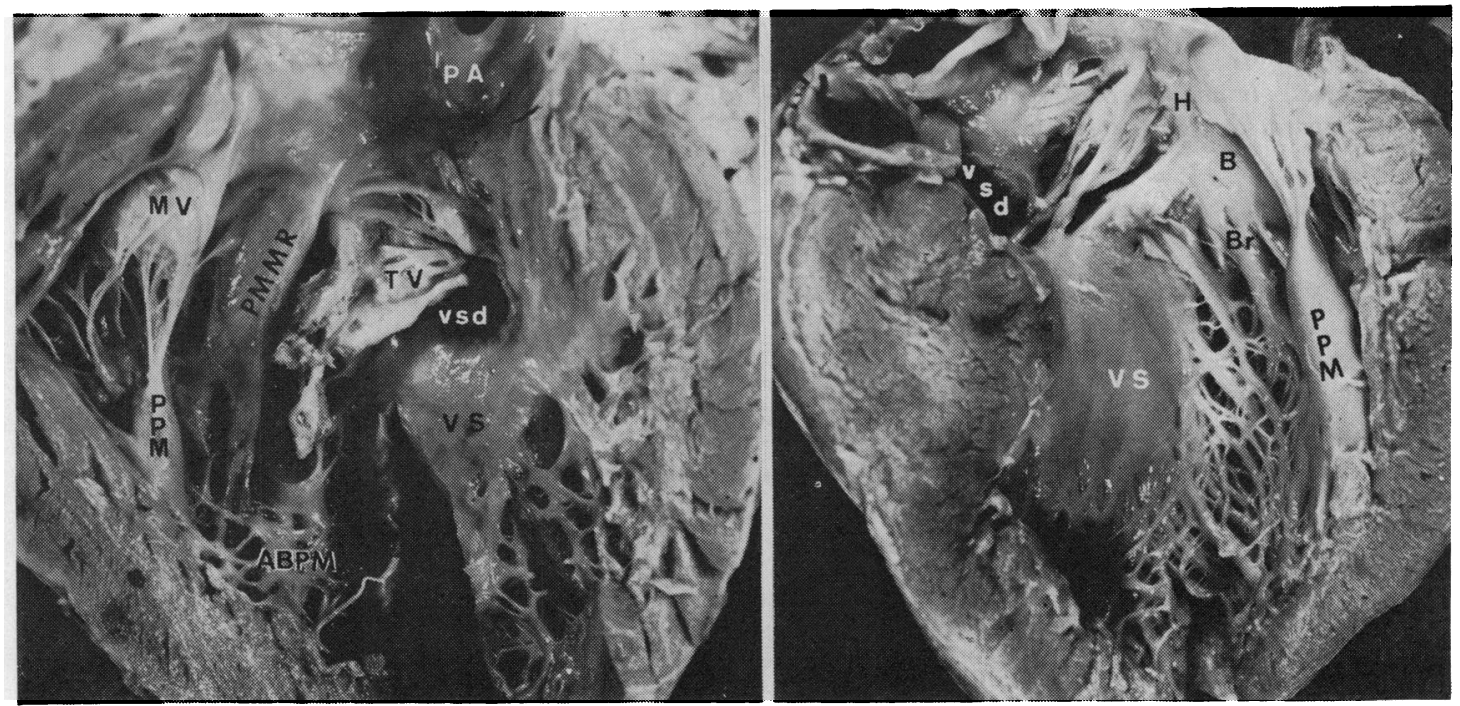

(A) Fig. 2 (A) Transposition with aorta to left and anterior to pulmonary artery $(P A)$ with inversion of ventricles and straddling tricuspid valve. In middle: junction of both $A V$ valves (TV and $M V$ ) with large PMMR protruding underneath into left ventricular cavity and branching toward base of posterior papillary muscle (PPM) and to right toward base of an abnormal papillary muscle $(A B P M)$ holding chordae of part of TV that lies in left ventricle. (B) Transposition with aorta to right and anterior to pulmonary artery and large posterior VSD: no muscular rim between tricuspid valve and edge of VSD. Head $(H)$ of posteromedian muscle ridge (PMMR) between both atrioventricular valve $(A V V)$ rings. Body $(B)$ of this muscle extending from septum to posterior free wall of left ventricle and its branches $(B r)$ joining base of PPM of left ventricle. 


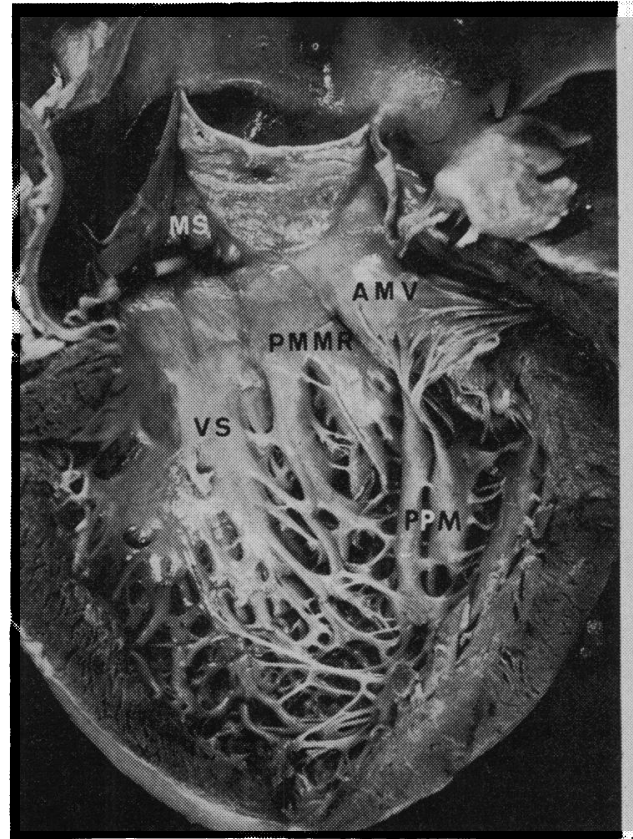

(A)

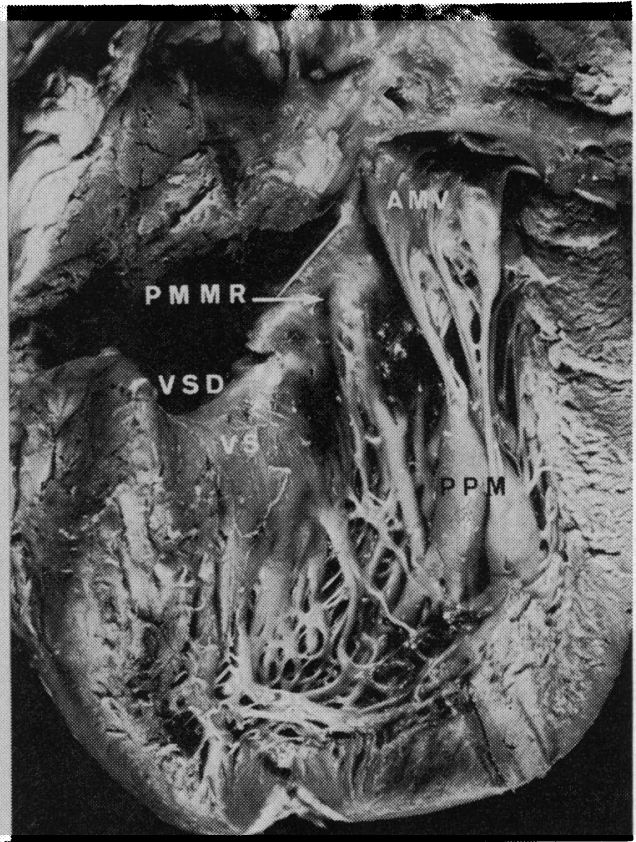

(B)

Fig. 3 Transposition with aorta to right and anterior to pulmonary artery with posterior (VSD). $(A)$ Under the membranous ventricular septum $(M S)$ a posteromedian muscle ridge (PMMR) protrudes on the ventricular septum (VS), extending parallel to the anterior mitral valve leaflets $(A M V)$ towards the base of the posterior papillary muscle (PPM) of the left ventricle. The body of the muscle splits up into two muscle bundles so that it looks like a double muscle body. (B) PMMR protrudes in an angle between VS and posterior left ventricular free wall. Head seems to be incorporated in posterior edge of VSD, while branches join base of PPM of left ventricle.

(Fig. 2 B). Its cranial insertion lies on the septum between the atrioventricular valves and its distal part goes toward the posterior wall of the left ventricle to reach the base of the papillary muscle.

The posteromedian muscle ridge could be identified in all cases with posterior ventricular septal defect and absence of a muscular rim between the tricuspid valve and the edges of the ventricular septal defect. In 14 of the 22 specimens the posteromedian muscle ridge was large. In some cases it was so large that it must have interfered, to some extent, with the flow of blood from the left ventricular body into the left ventricular outflow tract. The obstruction caused by such a ridge alone, however, may not be sufficient to produce a pressure gradient. It could add to the narrowing caused by other structures located more distally, such as the mitral valve apparatus and the anterolateral muscle described by Moulaert (1974).

In half of the specimens with a midseptal or a posterior ventricular septal defect with muscular rim between the tricuspid valve and the edge of the ventricular septal defect, a similar muscular structure was seen apically from the posterior margin of the membranous septum or its remnants (Fig. $3 \mathrm{~A})$. The head of the muscle seemed to be incorporated in the septum, but the body extended either on the septum (Fig. $3 \mathrm{~A}$ ) or into the angle made by the junction of the posterior wall and the ventricular septum (Fig. 3 B). The muscle continued parallel to and at the septal side of the anterior leaflet of the mitral valve (Fig. $3 \mathrm{~A}$ ). The branches also pointed to the posterior papillary muscle and sometimes joined it.

When the ventricular septal defect is purely anterior or when the ventricular septum is intact, this median muscle ridge is not prominent (Fig. $4 \mathrm{~A}$ ); it could be identified, however, in 21 per cent of our specimens.

In double inlet ventricle with left ventricular morphology and outlet chamber the posteromedian muscle ridge is also present and seems to anchor the atrioventricular valves to the posterior wall far away from the septum (Fig. 4 B). In a single case with common atrioventricular valve, a muscle similar to a posteromedian muscle ridge was found. It was 


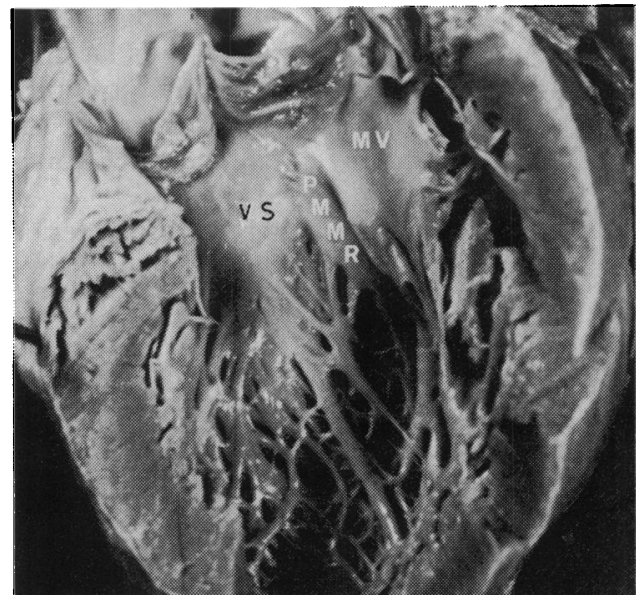

(A)

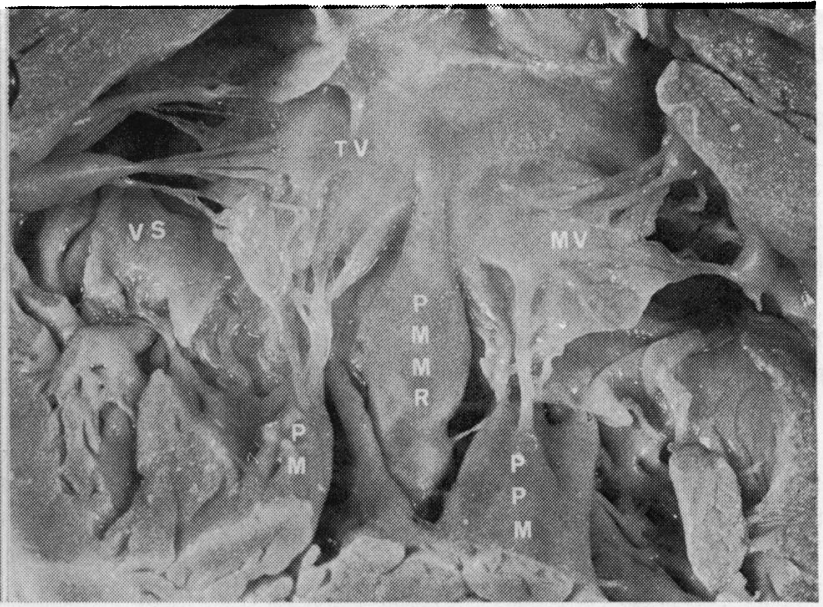

(B)

Fig. $4(A)$ Transposition with aorta to right and anterior to pulmonary artery and without ventricular septal defect (VSD). View of left VS, anterior mitral leaflet, and opened pulmonary artery. A small posteromedian muscle ridge $(P M M R)$ dissociates itself from the ventricular septum $(V S)$ and continues down along the mitral valve $(M V)$ in an angle between VS and posterior free wall toward base of posterior papillary muscle (PPM) of left ventricle. (B) Double inlet ventricle with aorta originating from outlet chamber right anterior of pulmonary artery. On the posterior ventricular free wall a PMMR extends from the junction of both $A V$ valves (TV and MV) to the base of the papillary muscle (PM), far away from the ventricular septum $(V S)$.

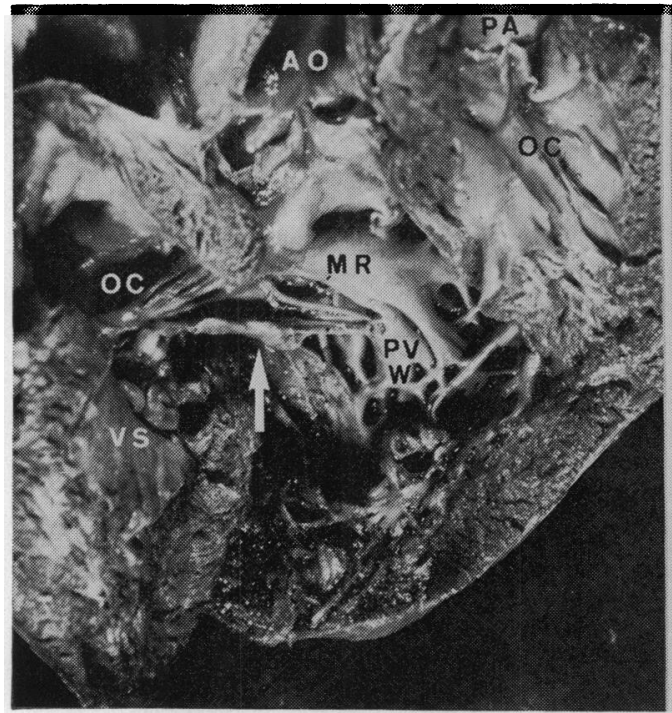

(A)

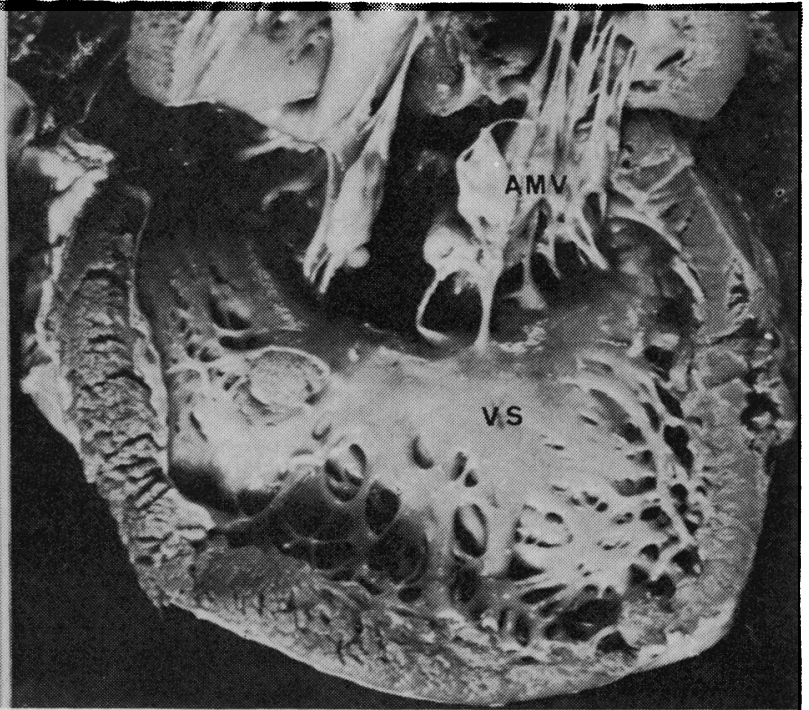

(B)

Fig. 5 (A) Double inlet ventricle with common atrioventricular valve (AVV) and left anterior outlet chamber (OC). Left, anterior part of ventricle, VS, and OC. Right, posterior part of ventricle and OC with pulmonary artery $(P A)$. In middle, common AV valve is stretched out (white arrow); cranially and in the centre of this valve a muscle ridge $(M R)$ is attached to it; from there the muscle ridge extends down the posterior free wall of the ventricle (PVW) to the base of the posterior papillary muscles. (B) Common atrioventricular canal. View of left side of ventricular septum (VS) and deformed anterior mitral valve (AMV). No median muscle ridge. 
related to the posterior cusp of the valve, anchoring it to the posterior wall of the left ventricle where the atrial septum emerged on the atrial side (Fig. $5 \mathrm{~A}$ ). From there it extended down toward the posterior papillary muscle. The anatomy in this case, however, was so bizarre that it was not possible to identify the muscle as the posteromedian muscle ridge.

In the 3 cases of double inlet ventricle with right ventricular morphology a small but distinct posteromedian muscle ridge was found distal to the junction of the two atrioventricular valve rings. It had a distorted aspect in the sense that head, body, and branches did not lie in a straight line but in a curved line that had its convexity toward the right. The branches did join the posterior papillary muscles of both atrioventricular valves. The head and part of the body of the posteromedian muscle ridge formed the right posteroinferior edge of the communication of the large ventricle with the diminutive chamber. The posteromedian muscle ridge seemed to be a good landmark for location of the entrance to this pouch.

Twenty-eight specimens of complete common atrioventricular canal were studied to ascertain the presence or absence of the muscle ridge when fusion of the atrioventricular cushions was lacking. It was not possible to identify any structure resembling the posteromedian muscle ridge in two-thirds of the hearts with complete common atrioventricular canal (Fig. 5 B).

On lifting up the posterior part of the anterior common leaflet in a third of the specimens, however, a small muscle ridge was identified extending from the atrioventricular valve ridge ring down the free wall of the left ventricle. This muscle had no distinct head, but its branches joined the posterior papillary muscle of the left ventricle (Fig. 6 B). In 2 cases a similar decapitated muscle ridge was present beneath the anterior part of the split anterior common leaflet as well (Fig. $6 \mathrm{~A}$ ). In none of our 28 cases was the so-called head of the posteromedian muscle ridge present.

\section{Discussion}

The fact that a posteromedian muscle ridge is not prominent in hearts without a ventricular septal defect or pure anterior ventricular septal defect, but is well developed in hearts in which it is sited away

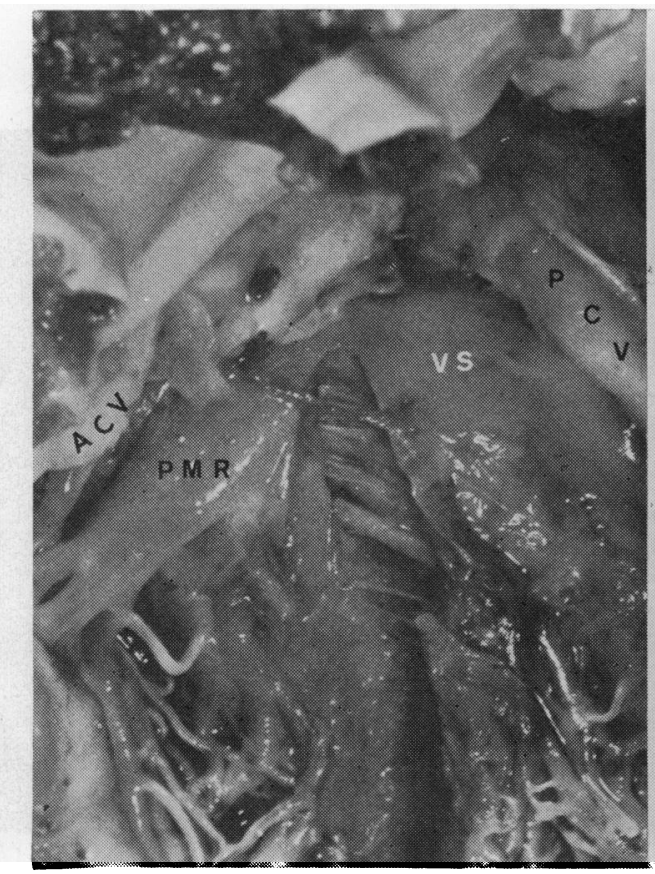

$(A)$

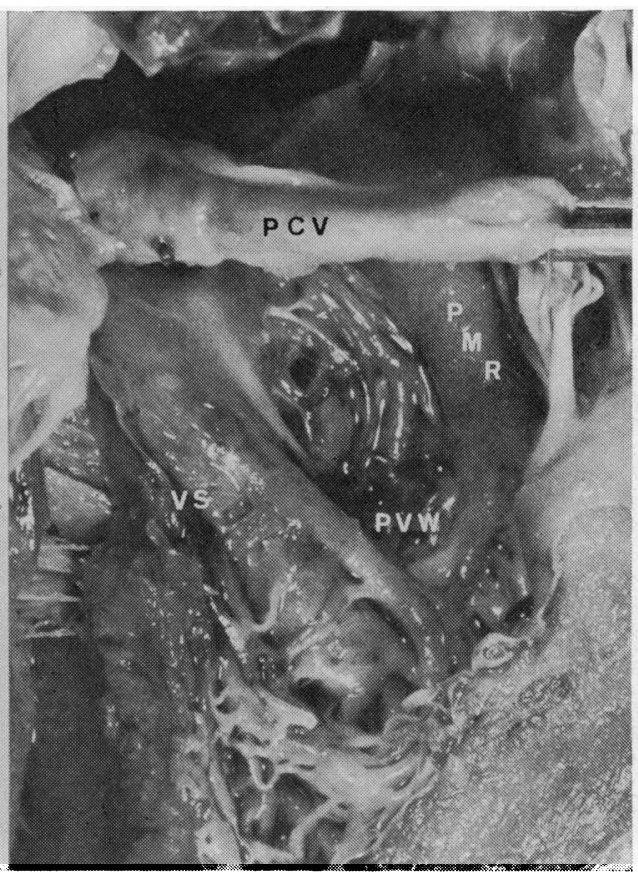

(B)

Fig. 6 Common atrioventricular canal. View of left side of ventricular septum (VS). (A) On lifting up anterior leaflet of common atrioventricular valve $(A C V)$ a muscle ridge $(P M R)$ is detected continuing down the anterior part of $V S$ to the base of the anterior papillary muscle of left ventricle. (B) Under posterior leaflet of common. atrioventricular valve (PCV) another muscle ridge (PMR) extends down the posterior free wall $(P V W)$ of the left ventricle joining the base of the posterior papillary muscle of the left ventricle. 
from the septum suggests that the flow, entering the left ventricle on both sides of the posteromedian muscle ridge, creates a favourable situation for the muscle body to develop by a process of diverticulation and undermining in the same way as suggested for the development of papillary muscles (Netter, 1969).

The head of the posteromedian muscle ridge maintains a constant relation with the junction of the two atrioventricular valve rings even if this junction is sited away from the ventricular septum on the posterior left ventricular wall or in the right ventricle. If the septum separates the valves, the head seems to be part of the septum located just apical and posterior from the septum membranaceum (Fig. $3 \mathrm{~A}$ ). The cranial insertion of the posteromedian muscle ridge is always situated where the atrial septum joins the central fibrous body.

On comparing specimens of transposition without ventricular septal defect (Fig. $4 \mathrm{~A}$ ) or with anterior ventricular septal defect (Fig. $3 \mathrm{~A}$ ) and those with posterior ventricular septal defect (Fig. $2 \mathrm{~B}$ ), it is noted that the posteromedian muscle ridge detaches itself from the septum and becomes more and more prominent as the defect becomes more posterior. In specimens with posterior ventricular septal defect in which the muscular rim between the edge of the ventricular septal defect and the tricuspid valve is missing, it is difficult to determine whether or not the tricuspid valve is straddling the septum (Fig. $2 \mathrm{~B})$. In these instances the posteromedian muscle ridge glides off the septum into the left ventricle, taking the tricuspid valve with it. The straddling of this valve becomes more and more obvious (Fig. 2 A). Finally, there is only a small part of the right atrioventricular valve left in the remaining part which we can no longer term a 'right ventricle' because it has practically no inlet (Fig. 7). This is the last step toward the formation of the anomaly of double inlet ventricle of the left ventricular type with outlet chamber (Fig. 4 B).

One can speculate that normal migration of the bulboventricular septum toward the atrioventricular canal during normal development follows the opposite direction, as we have described. Then the separation of the posteromedian muscle ridge from the ventricular septum means a splitting up of the ventricular septum into the derivatives of bulboventricular septum and the derivatives of atrioventricular canal septation. This hypothesis is reinforced by the fact that in the complete atrioventricular canal where such septation did not occur, the head of the muscle was not found in any of the 28 specimens.

The cases of double inlet ventricle of the right ventricular type could be the end point of another spectrum, that of the straddling of the mitral valve over the ventricular septum. Here the derivates of atrioventricular canal septation and ventricular septum do meet and even pass each other so that both atrioventricular valve rings and the posteromedian muscle ridge are in the right ventricle.

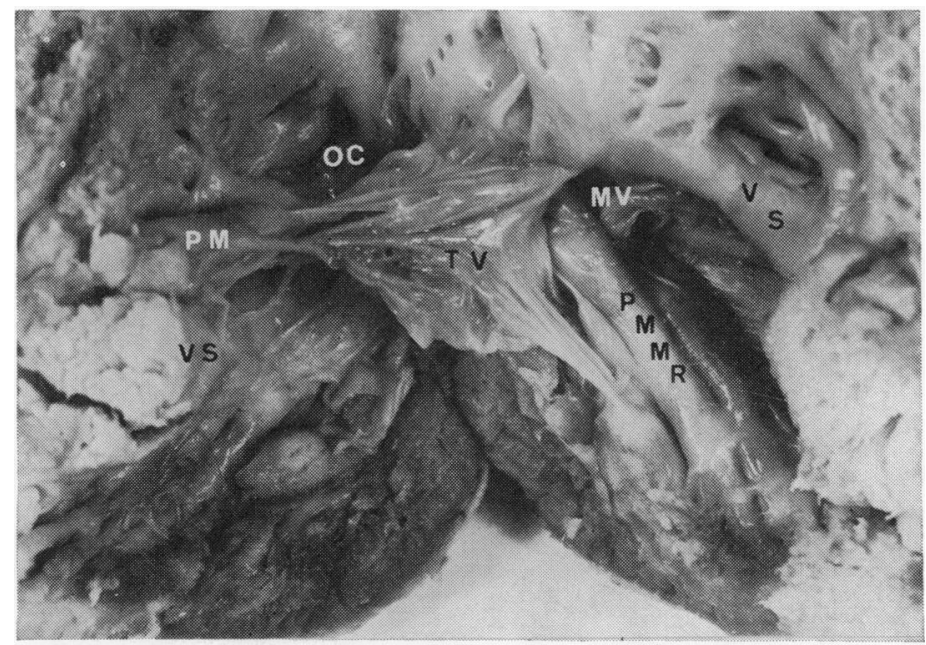

Fig. 7 Double inlet ventricle with hypoplastic mitral valve (MV), left anterior outlet chamber, and pulmonary valve atresia. Left, anterior part of ventricle, ventricular septum (VS), and outlet chamber (OC). Right, posterior part of ventricle, VS, and OC; hypoplastic MV and PMMR between TV and $M V$. Centre, large tricuspid valve with papillary muscle joining PMMR; another papillary muscle $(P M)$ crosses $V S$ and is attached to the free wall of $O C$. 
In double inlet ventricle with outflow chamber, the muscle ridge between the two atrioventricular valves demarcates the two inflow tracts and is an ideal ridge on which to suture the septating patch. It has recently been shown that this ridge does not carry the conduction tissue in double inlet ventricle with an outflow chamber (Anderson et al., 1974; Maloney et al., 1975); suturing in this region, therefore, is a safe procedure.

The authors thank Dr A. Oppenheimer-Dekker and the staff members of the Department of Embryology and Anatomy of the University of Leiden, Holland, for giving them the opportunity to study 21 specimens from their anatomical collection. They are also grateful to Dr A. Oppenheimer and to Dr A. Moulaert of Utrecht, Holland, for their invaluable advice in identifying certain anatomical structures in some of the specimens included in this study.

\section{References}

Anderson, R. H., Arnold, R., Thapar, M. K., Jones, R. S., and Hamilton, D. I. (1974). Cardiac specialized tissue in hearts with an apparently single ventricular chamber (double inlet left ventricle). American fournal of Cardiology, 33, 95-106.

De la Cruz, M. V., and Miller, B. L. (1968). Double-inlet left ventricle: two pathological specimens with comments on the embryology and on its relation to single ventricle. Circulation, 37, 249-260.

Goor, D. A., and Edwards, J. E. (1973). The spectrum of transposition of the great arteries, with specific reference to developmental anatomy of the conus. Circulation, 48, 406415.

Liberthson, R. R., Paul, M. H., Muster, A. J., Arcilla, R. A., Eckner, F. A. O., and Lev, M. (1971). Straddling and displaced atrioventricular orifices and valves with primitive ventricles. Circulation, 43, 213-226.

Maloney, J. D., Ritter, D. G., McGoon, D. C., and Danielson, G.K. (1975). Identification of the conduction system in corrected transposition and common ventricle at operation. Mayo Clinic Proceedings, 50, 387-394.

Mehrizi, A., McMurphy, D. M., Ottesen, O. E., and Rowe, R. D. (1966). Syndrome of double inlet left ventricle: angiocardiographic differentiation from single ventricle with rudimentary outlet chamber. Bulletin of the fohns Hopkins Hospital, 119, 255-267.

Moulaert, A. (1974). Ventricular Septal Defect and Anomalies of the Aortic Arch. Luctor and Emergo, Leiden.

Netter, F. H. (1969). The Ciba Collection of Medical Illustrations. Vol. 5: Heart, Section III. Embryology, pp. 111-130. Ciba Pharmaceutical Products, Summit, New Jersey.

Rastelli, G. C., Ongley, P. A., and Titus, J. L. (1968). Ventricular septal defect of atrioventricular canal type with straddling right atrioventricular valve and mitral valve deformity. Circulation, 37, 816-825.

Tandon, R., Becker, A. E., Moller, J. H., and Edwards, J. E. (1974). Double inlet left ventricle straddling tricuspid valve. British Heart fournal, 36, 747-759.

Wenink, A. C. G., Oppenheimer-Dekker, A., Van Gils, F. A. W., Gittenberger-de Groot, A. C., and Moulaert, A. J. M. G. (1976). Transposition of the great arteries: embryological considerations (abstract). Seventh European Congress of Cardiology, I, p. 33.

Requests for reprints to $\mathrm{Dr}$ Anne DevlooBlancquaert, Akademisch Ziekenhuis, 9000 Gent, Belgium. 